\title{
Targeting Fe-S Protein to Fight Neglected Tropical Diseases
}

\author{
P Feliciano ${ }^{1}$, C Drennan ${ }^{2}$ \\ ${ }^{1}$ MIT/HHMI, Cambridge, MA, ${ }^{2}$ MIT \\ pattyrf@mit.edu
}

Here we describe a novel [4Fe-4S] cluster-containing metalloprotein: cytosolic fumarate hydratase from Leishmania major (LmFH-2). Like aconitase, it uses a [4Fe-4S] cluster to coordinate substrate but is not structurally similar to aconitase. In fact, $\mathrm{LmFH}-2$ has a unique protein fold. Importantly, metal-dependent FHs are found in parasites that cause Neglected Tropical Diseases (NTD), such as leishmaniases, Chagas disease, and sleeping sickness, whereas humans employ a metal-independent FH. NTDs affect approximately one-sixth of the world's population, and due to the appearance of resistance to current medicines, there is an increased interest in the development of innovative strategies to combat NTD. To explore the potential of FHs as anti-NTD targets, we have determined a series of structures of Leishmania major FH, including a structure with the substrate malate bound to the unique iron of the cluster. Our structures provide insights into the catalytic mechanism of class I (metaldependent) FHs, a mechanism that is likely to be conserved among FHs from NTD-causing organisms.

Acta Cryst. (2020). A76, a98 